\title{
Efectos pandemia en la formación quirúrgica: visión del residente de cirugía general
}

\author{
Pandemic Effects on Surgical Training: General Surgery Resident's View
}

Deycies Gaete L. ${ }^{1,2}$

La enfermedad por Coronavirus 2019 (COVID-19), identificada por primera vez en Wuhan, China en diciembre de 2019, se ha convertido en una pandemia mundial con impacto en todas las esferas del desarrollo humano, afectando directamente las profesiones de la salud y, en particular, las del área quirúrgica, como cirugía general y las especialidades derivadas.

Sumado a lo anterior, ya desde el 2008, Hepp y cols. ${ }^{1}$, identificaron situaciones tales como cambios epidemiológicos, aumento de la especialización de la Cirugía, incorporación de nuevas tecnologías, judicializacion del desempeño de la medicina, incorporación de la Medicina Basada en Evidencia, y, ausencia de estandarización de los programas, que han incidido en una disminución de la exposición a oportunidades quirúrgicas para los residentes. Por lo tanto, si previo a la pandemia la formación de becados ya enfrentaba adversidades, esta última ha venido a agudizar dicha situación.

Para objetivar la percepción de la disminución de la actividad quirúrgica se ha consultado los registros nacionales del Departamento de Estadística e Información de Salud (DEIS) del Ministerio de Salud, encontrando una disminución progresiva de las hospitalizaciones electivas por hernias y colelitiasis, desde el 2017, con un quiebre importante entre 2019 y 2020 (Figura 1). Tal fenómeno no se observa de la misma magnitud en la incidencia de hospitalizaciones por apendicitis aguda, concluyendo que el mayor impacto en la merma de las oportunidades quirúrgicas producto de la pandemia se ha dado en la cirugía electiva.

Actualmente se imparten 25 Programas de Cirugía General (60\% en la Región Metropolitana), con más de 280 becarios en formación. Si bien Uribe y cols $^{2}$ ya analizaron los efectos de la pandemia en la educación de los futuros cirujanos, para complementar esto con la visión del residente, la Directiva de la Seccional de Residentes de la Sociedad Chilena de Cirujanos ejecutó una encuesta on-line a una muestra de 211 residentes de Cirugía General, de los distintos programas y años de formación del país, entre diciembre de 2020 y enero de 2021, cuyos resultados se describen en la Tabla 1 . Como percepción general, la pandemia ha mermado la oportunidad quirúrgica de aprendizaje "in vivo", incluso provocando la perdida de rotaciones consideradas esenciales para la adquisición de competencias de un cirujano general. Más aún, no debemos olvidar, que el COVID no solo afectó la educación quirúrgica, sino también, el estado de salud, físico y mental, familiar y personal, de los resientes, presentandose enfermedad por SARS-CoV-2 en un $69 \%$ de los encuestados, generando pérdidas por discapacidad temporal, hospitalización, rehabilitación y diversos efectos secundarios en el bienestar biopsicosocial de los becarios, además de ser requeridos por sus respectivos Servicios de Salud para apoyar las labores asistenciales de sus centros de origen.

Es dentro de este contexto, en donde adquiere vital importancia el desarrollo de Centros de Simulación Quirúrgica, básicos y avanzados, en técnicas abiertas y mínimamente invasivas, como complemento a las herramientas educativas de los Programas de Cirugía General, permitiendo; crear entornos de entrenamiento seguro, ejercitación de todo tipo de habilidades, desarrollar programas de formación individualizados, acelerar el proceso de aprendizaje, acortando el número de procedimientos requeridos para considerar a una técnica como aprendida, la realización de feedback y debriefing, el entrenamiento interprofesional y la estandarización del aprendizaje ${ }^{3}$.

Cabe destacar el rol activo protagonizado por el Departamento de Educación de la Sociedad de Cirujanos de Chile, quien ha efectuado más de 20 exposiciones virtuales gratuitas para los residentes de cirugía, demostrando preocupación por le formación de futuros colegas.
Residente de Cirugía General, Departamento de Cirugía Hospital Clínico de Universidad de Chile. 2 Presidente Departamento de Residentes Sociedad de Cirujanos de Chile.

Correspondencia a: Dra. Deycies Gaete L. dey.gaete@gmail.com 


\section{EDITORIAL}

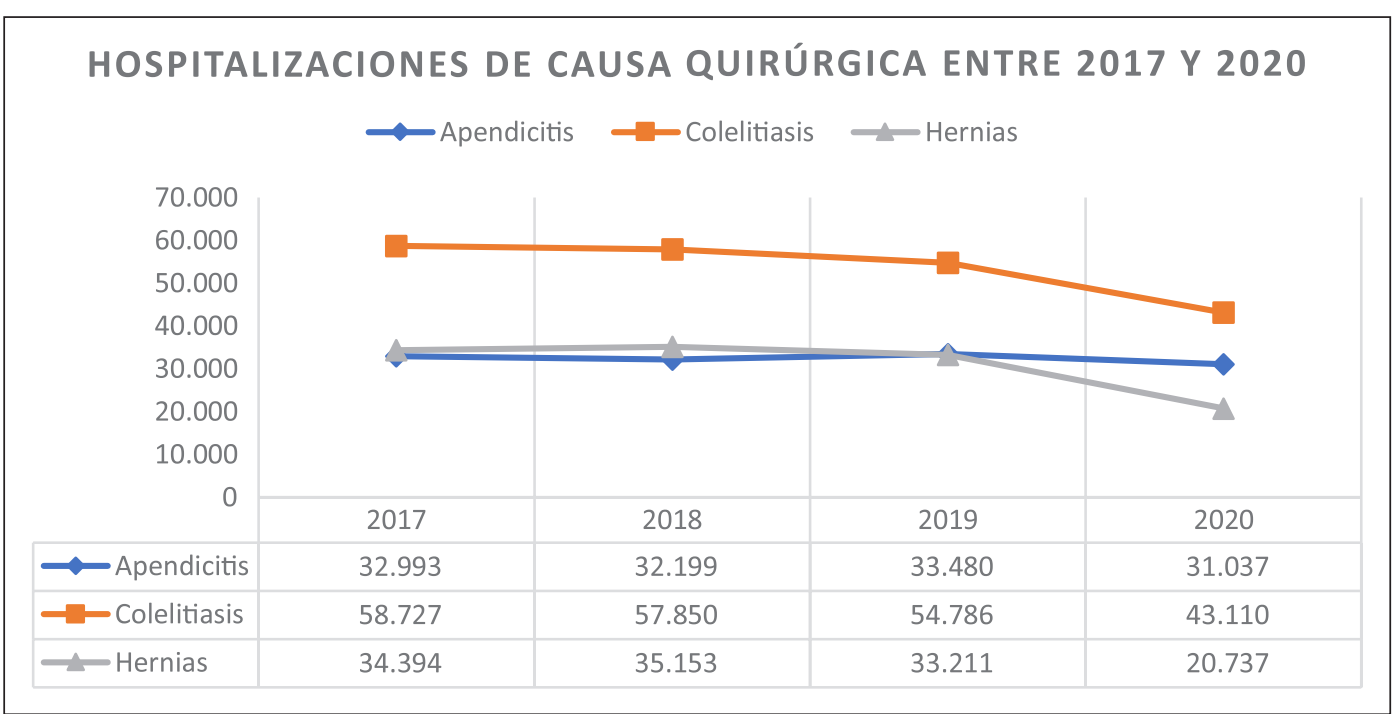

Figura 1. Hospitalizaciones Nacionales de colelitiasis, hernias y apendicitis aguda entre 2017 y 2020 Fuente: https://deis.minsal.cl/

Tabla 1. Resultados de Encuesta on-line a Residentes de Cirugía General respecto a la percepción de los daños causados por la pandemia en su formación

\begin{tabular}{|c|c|}
\hline Ámbito & Resultado \\
\hline Disminución de la Actividad Quirúrgica docente & $90,4 \%$ \\
\hline Prolongación de los meses de Beca & $52,1 \%$ \\
\hline Perdida de rotaciones & $53,2 \%$ \\
\hline Residentes con COVID (+) & $44,3 \%$ \\
\hline Residentes catalogados como contacto estrecho & $13,7 \%$ \\
\hline Residentes sometidos a cuarentena de al menos 14 días & $69 \%$ \\
\hline
\end{tabular}

En conclusión, la pandemia ha generado importantes repercusiones en las oportunidades de aprendizaje asistencial en la formación de los residentes, principalmente en cirugías programadas, $\mathrm{y}$, aún más importante, en el bienestar psicosocial de los mismos y sus familias. Dentro de las estrategias que propo- nemos para mermar los daños de la pandemia están; fomentar la implementación de Centros de Simulación como elemento complementario para adquirir habilidades técnicas, estandarización de los programas de formación, y, finalmente, la definición de las competencias y perfil de egreso del cirujano general.

\section{Bibliografia}

1. Hepp KJ, Csendes JA, Ibáñez CF, Llanos LO, San Martín RS.

Programa de la especialidad Cirugía General: Definiciones y propuestas de la Sociedad de Cirujanos de Chile.
Rev Chil Cir. 2008; 60:79-85.

2. Uribe MM, Martínez SG, Sepúlveda DR, Lanzarini SE, García CC, Cárcamo IC, et al. Efectos de la pandemia en los programas de formación en cirugía. Problemas y soluciones. Rev Cir. 2021;73:107-13.
3. Ruiz-Gómez JL, Martín-Parra JI, González-Noriega M, RedondoFiguero CG, Manuel-Palazuelos JC. La simulación como modelo de enseñanza en cirugía. Cirugía Española 2018;96:12-7. doi:10.1016/j. ciresp.2017.09.005. 